\title{
THE DISTRIBUTION OF GALACTIC DISK STARS IN BAADE'S WINDOW
}

\author{
B. Paczyński, K. Z. Stanek ${ }^{1}$ \\ Princeton University Observatory, Princeton, NJ 08544-1001 \\ A. Udalski, M. Szymański, J. Kałużny, M. Kubiak \\ Warsaw University Observatory, Al. Ujazdowskie 4, 00-478 Warszawa, Poland \\ and \\ M. Mateo \\ Department of Astronomy, University of Michigan, 821 Dennison Bldg., Ann Arbor, \\ MI 48109-1090
}

\begin{abstract}
The color-magnitude diagram of $\sim 3 \times 10^{5}$ stars obtained for Baade's Window towards the Galactic bulge with the OGLE project reveals a surprisingly narrow main sequence due to galactic disk stars at a distance of $d \sim 2 \mathrm{kpc}$, i.e. at the location of the Sagittarius spiral arm. A more careful analysis indicates there is an excess in the number of disk stars by a factor $\sim 2$ between us and $d \sim 2.5$ $\mathrm{kpc}$ and a rapid drop by a factor $\sim 10$ beyond that distance. It is unlikely that the observed structure is an artifact of the interstellar extinction, but careful determination of the extinction is needed before the structure is firmly established.

The narrow main sequence extends down to stars as faint as $M_{V} \approx 7$, i.e. it is composed of old stars. This is not expected in a conventional disk model, or in a conventional model of a spiral structure. However, a strong concentration of old stars towards spiral arms has been noticed in some other galaxies, like M51 (Rix \& Rieke 1993), with the near infrared surface photometry.

We have also found that the relative distribution of red clump and red giant stars in the Galactic bulge implies that there is a relatively young stellar population present there. Color-magnitude diagram data is accessible over the computer network with anonymous ftp.
\end{abstract}

Subject headings: stars: HR diagram - stars: statistics - galaxy: general galaxy: structure

\footnotetext{
${ }^{1}$ On leave from N. Copernicus Astronomical Center, Bartycka 18, Warszawa 00-716, Poland
} 


\section{INTRODUCTION}

The Optical Gravitational Lensing Experiment (OGLE, Udalski et al. 1992, 1993b) is an extensive photometric search for the rare cases of gravitational microlensing of the Galactic bulge stars by the Galactic disk stars, brown dwarfs and planets. It provided a huge data base for the studies of other problems (Szymański \& Udalski 1993), in particular the color-magnitude diagrams (Udalski et al. 1993a). These revealed an expected population of bulge stars, with its turn-off point, red giant branch and red clump, but also an unexpected concentration of stars in the blue part of the color-magnitude diagrams. This feature may be attributed to foreground disk stars, which seem to concentrate at a single distance, possibly coinciding with the Sagittarius spiral arm. This concentration in the color-magnitude diagram was previously noticed by Terndrup (1988) and Tyson (1991), and even earlier by Rodgers, Harding \& Ryan (1986), but it shows up much more clearly in our data (Udalski et al. 1993a), because of unprecedented coverage combined with very good seeing. It seems that the full consequences for the distribution of the Galactic disk stars were not appreciated in the past, even though Rodgers et al. (1986) and Terndrup (1988) noticed that the observed number of disk stars is approximately twice as large as expected in a standard model, and a possible association with the spiral arm was briefly mentioned by Terndrup (1988) and by Udalski et al. (1993a). In this paper we present the

summary of our observational results, we confront them with the expectations based on the standard Galaxy model (Bahcall \& Soneira 1980, hereafter B\&S; Bahcall 1986), and we make tentative suggestions about the meaning of the apparent conflict.

\section{THE DATA}

Udalski et al. (1993a) present color-magnitude diagrams (CMDs) of 14 fields in the direction of the Galactic bulge, which cover nearly one square degree and contain about $6 \times 10^{5}$ stars. All observations were made using the 1 meter Swope telescope at the Las Campanas Observatory, operated by the Carnegie Institution of Washington, and a $2048 \times 2048$ pixel Ford/Loral CCD detector with the pixel size 0.44 arcsec covering $15^{\prime} \times 15^{\prime}$ field of view. In this paper we discuss only the nine partly overlapping fields in Baade's Window, covering a total area of $40^{\prime} \times 40^{\prime}$ and centered at $b=-3.9^{\circ}, l=1.0^{\circ}$. As an example the CMD for the central field (BWC) is shown in Figure 1, together with five straight lines which provide a schematic representation of the location of disk main sequence stars at the distance of $0.5,1,2,4$, and $8 \mathrm{kpc}$, with the interstellar extinction adopted 
following $\operatorname{Arp}\left(1965\right.$, cf. eqs. 3 in this paper, with $\left.A_{V, G C}=1.5\right)$, and the $(V-I)_{0}$ color calculated with the eq. (11) of this paper. Most of the diagram is dominated by the bulge stars, with the disk stars concentrated mostly near the line corresponding to $\sim 2 \mathrm{kpc}$. The part of the diagram dominated by the disk stars is shown in Figure 2, displaying all stars from the nine fields in Baade's Window and the same five main sequence lines, as well as four solid lines corresponding to the disk main sequence stars of the absolute visual magnitude and the unreddened $V-I$ color: $\left[M_{V},(V-I)_{0}\right]=(1.0,0.0),(3.0,0.2),(5.0,0.4)$, and $(7.0,0.6)$, respectively (cf. section 3$)$. There are $\sim 16,000$ stars plotted in Figure 2, and we believe the most of them are in the Galactic disk.

The distribution of stars in Figure 2 is very surprising. A conventional B\&S model of the Galactic disk has the number density of stars decreasing exponentially with the distance from the Galactic plane with the scale height of $\sim 300 \mathrm{pc}$, and increasing exponentially towards the Galactic center with the length scale of $\sim 3.5 \mathrm{kpc}$. These conspire to give the number density of stars which is approximately constant with distance while looking through Baade's Window, i.e. it implies from geometry that we expect the number of stars to increase by a factor of $\sim 4$ per magnitude. This is approximately what is observed out to the distance of $\sim 2 \mathrm{kpc}$, but there is a dramatic deficit in the number of stars at larger distances. There was evidence for this sequence of the Galactic disk stars in the CMDs obtained previously for Baade's Window as well as for other areas - further away from the Galactic plane: $(l, b) \approx\left(0^{\circ},-25^{\circ}\right)$ (Rodgers et al. 1986), $(l, b) \approx\left(0^{\circ},-8^{\circ}\right)$ (Terndrup 1988) - and closer to the Galactic plane: $(l, b) \approx\left(1^{\circ},-2^{o}\right)$ (Tyson 1991), and $(l, b) \approx\left(14^{o},-1^{\circ}\right)$ (Ortolani, Bica \& Barbuy 1992), but the sequence shows much more clearly in our data.

The interstellar extinction toward Baade's Window merits some discussion. There are many estimates of extinction towards the globular cluster NGC 6522 located near the center of our central field BWC (Arp 1965; Walker \& Mack 1986; Terndrup 1988; Walker \& Terndrup 1991; and references therein); their estimates of extinction towards NGC 6522 range from $A_{V}=1.5$ all the way to $A_{V}=1.78$. We adopt $A_{V, B W C}=1.5$ as a reasonable value (Arp 1965, Terndrup 1988) for our initial presentation, but we explore a large range of values in our discussion (section 4).

We estimate the extinction towards the Galactic bulge in our other eight fields as follows. We selected the red giants according to the following conditions:

$$
1.5<V-I<2.4, \quad 13.0<V_{V-I} \equiv V-2.6(V-I)<14.5,
$$

and the red clump stars according to

$$
1.5<V-I<2.4, \quad 11.5<V_{V-I} \equiv V-2.6(V-I)<13.0,
$$


in all nine fields. There were about 3,000 red giant stars and 4,000 red clump stars in every field. The parameter $V_{V-I}$ has been chosen so that for any particular star its value is not affected by the unknown extinction. The distribution of $V-I$ colors was determined for each group in every field. The forms of the distributions were similar except for a small shift in the $V-I$ color caused by the differences in extinction between the fields. The results are given in Table 1: column 1 provides the name of the field, columns 2 and 3 give the galactic coordinates of the field center, columns 4 and 5 give the difference $\Delta(V-I)$ between a given field and BWC as obtained with the red giants (rg) and the red clump stars (rc), respectively, column 6 gives the total visual absorption adopted for each field, $A_{V, G B}$, and the last column contains the value of the $V_{V-I}$ parameter at which the distribution of the red clump stars has its maximum. With an rms difference of 0.03 mag, it is clear that the two measures of $\Delta(V-I)$ agree with each other quite well. The average value of extinction for all 9 fields covering the OGLE Baade Window is larger then that measured towards NGC 6522 by $\left\langle\Delta A_{V, G B}\right\rangle=0.09$. A reasonable range for the average value of total extinction is $1.6 \leq\left\langle\Delta A_{V, G B}\right\rangle \leq 1.9$.

While the total extinction towards the Galactic bulge seems to be fairly well established the variation of extinction with distance along the line of sight is poorly known. Following Arp (1965) we adopt the following simple law:

$$
\begin{array}{ll}
A_{V}=A_{V, G B} \times(d / 2 k p c) & \text { for } \quad d<2 k p c \\
A_{V}=A_{V, G B} & \text { for } \quad d>2 k p c,
\end{array}
$$

and $E_{V-I}=A_{V} / 2.6$, following Dean, Warren, \& Cousins (1978) and Walker (1985).

Notice that according to Table 1 the distribution of the $V_{V-I}$ parameter peaks at the value of $12.36 \pm 0.03$ in all nine fields, indicating that it is almost unaffected by the interstellar extinction, as designed. However, there is some correlation between $A_{V, G B}$ and $V_{V-I}$ as given in Table 1 , indicating that our choice of the ratio $A_{V} / E_{V-I}=2.6$ was not perfect.

\section{THE STANDARD MODEL}

We calculated the expected distribution of foreground Galactic disk stars following the standard B\&S model adopting disk parameters as given by Bahcall (1986, p. 591). The number density of disk stars $n_{D}$ per cubic parsec is given as

$$
n_{D}=n_{D}\left(R_{0}\right) \exp \left[-|z| / H\left(M_{V}\right)\right] \exp \left[-\left(R_{G C}-R_{0}\right) / h\right]
$$


where $R_{G C}$ is the galactocentric distance, $\mathrm{z}$ is the distance from the Galactic plane, $n_{D}\left(R_{0}\right)$ is the star number density near the sun, i.e. at the distance $R_{0}=8 \mathrm{kpc}$ (Holtzman et al. 1993 and references therein) from the Galactic center, $H\left(M_{V}\right)$ is the scale height of the distribution of stars of absolute visual magnitude $M_{V}$, and $h=3.5 \mathrm{kpc}$ is the disk scale length. The scale height $H\left(M_{V}\right)$ is given by Bahcall (1986, p. 595) as

$$
\begin{array}{llcc}
H\left(M_{V}\right)=90 p c & \text { for } & M_{V}<+2.3, \\
H\left(M_{V}\right)=90 p c+83.9 p c\left(M_{V}-2.3\right) & \text { for } & +2.3<M_{V}<+5.1, \\
H\left(M_{V}\right)=325 p c & \text { for } & +5.1<M_{V} .
\end{array}
$$

The disk luminosity function adopted by Bahcall is based on Wielen (1974). For stars with $M_{V}<+6$ it is almost identical with the Luyten (1968) luminosity function, for which Bahcall and Soneira (1980, p. 77) give analytical approximation:

$$
\Phi\left(M_{V}\right)=4 \times 10^{-3}\left[p c^{-3}\right] \frac{10^{0.04 x}}{\left(1+0.1^{0.206 x}\right)^{3.4}}, \quad x \equiv M_{V}-1.28 .
$$

Combining eqs. (1-3) we can calculate the number density of stars as a function of distance as seen through Baade's Window. In particular, we have the relations

$$
z=d \times \sin |b| \approx 0.068 d, \quad R_{G C}=\left|d-R_{0}\right|,
$$

where $\mathrm{d}$ is the distance from us, and we made an approximation $\cos b \approx 1$.

Notice that at the center of Baade's Window the eq. (4) can be written as

$$
\begin{array}{r}
n_{D, B W}=n_{D}\left(R_{0}\right) \exp \left[(d / 3.5 k p c)\left(1-238 p c / H\left(M_{V}\right)\right)\right], \\
\text { for } b=-3.9^{\circ}, \quad d<R_{0}=8 k p c,
\end{array}
$$

i.e. for stars of $M_{V} \approx 4$ the number density is constant with distance, for the brighter stars the number density is falling with distance, and for the fainter stars it is increasing with distance. The numerical coefficients vary across Baade's Window, but only a little.

The area of Baade's Window covered by the OGLE is $\left(40^{\prime}\right)^{2}$ which is $1.35 \times 10^{-4} \mathrm{rad}^{2}$. Therefore, the volume contained between distances $d_{1}$ and $d_{2}$ can be calculated as

$$
V_{1,2}=4.5 \times 10^{-5}\left(d_{2}^{3}-d_{1}^{3}\right)
$$

The empirical color-magnitude relation for the Pleiades main sequence was given by Walker (1985) and Prosser, Stauffer \& Kraft (1991). To this we apply a simple analytical fit:

$$
(V-I)_{Z A M S} \approx 0.2 \times\left(M_{V}-1.5\right), \quad 1.5<M_{V}<9.0
$$


which gives the rms deviation of only $0.05 \mathrm{mag}$ in V-I. The majority of disk stars are somewhat evolved. According to Castellani, Chieffi \& Straniero (1992) the width of the hydrogen burning main sequence is $\Delta M_{V} \approx 1.0 \mathrm{mag}$ at a fixed color. Therefore, we adopted the following approximate color-magnitude relation for the disk stars

$$
(V-I)_{D} \approx 0.2 \times\left(M_{V}-1.0\right), \quad 1.0<M_{V}<8.5
$$

The apparent magnitude and color are given as

$$
V=M_{V}+A_{V}(d)+5 \log (d / 10 p c), \quad V-I=(V-I)_{D}+E_{V-I}(d) .
$$

We crudely allowed for the spread of the main sequence stars in magnitude by adding to each value of $\mathrm{V}$ a random number with a uniform distribution in the range from -0.5 to +0.5 .

We have built our model as a sum of nine fields with the galactic coordinates and the value of total extinction listed in Table 1 , and the volume within each field adopted as 1/9 of that given with the eq. (9). Combining the equations presented in this chapter we used a Monte-Carlo simulation to make Figure 3, which presents the distribution of the main sequence disk stars expected to be seen in Baade's Window field of $\left(40^{\prime}\right)^{2}$. It is very different from what is observed (cf. Figure 2). In the standard model most stars are expected to be at the distance $d>3 k p c$, while in fact about $90 \%$ of the stars are observed to be at $d<3 k p c$. A possible explanation of this discrepancy is discussed in the next section.

Notice that our star counts were done in each of the nine fields as if there was no overlap, i.e. we did not identify stars visible in more than one field, hence some were counted more than once. There is approximately $15 \%$ overlap between the fields, so we have over-counted by a factor $\sim 1.15$. On the other hand our counts for stars brighter than $I=18.5$ are only $\sim 80 \%$ complete (Udalski et al. $1993 \mathrm{a}$, Table 4 , Sample \#1). The two effects almost cancel each other, and we make no correction for them. Our counts are likely to be accurate to $\sim 10 \%$, i.e. uncertainties in stellar counts are much smaller than the effects we describe in this paper.

\section{DISCUSSION}

The first modification of the standard model is suggested by the recent paper by Kent, Dame \& Fazio (1991) who find that the scale height of the near infrared disk light is 247 pc near us, i.e. at $R_{0}=8 \mathrm{kpc}$, but it is only $165 \mathrm{pc}$ at the galactocentric distance $R_{G C}=5$ 
kpc. We modify the standard B\&S model by multiplying the scale height as given with the eqs. (5) by a factor $\left(1-0.89 R_{G C} / R_{0}\right)$ :

$$
H\left(M_{V}, R_{G C}\right)=H\left(M_{V}, R_{0}\right) \times\left(1-0.89 \frac{R_{G C}}{R_{0}}\right) .
$$

Retaining all other details of the model unchanged the new distribution of stars in the CMD was calculated and it is shown in Figure 4. A visual impression is that the new distribution is closer to the data, but it is still not a good match.

To make a quantitative comparison between various models and the data it is useful to present the distribution of stars as a function of a parameter related to the distance modulus. The distance modulus can be expressed as

$$
\mu \equiv 5 \log (d / 10 p c)=V-5(V-I)-1.0+\left[5 E_{V-I}(d)-A_{V}(d)\right],
$$

(cf. eqs. 11 and 12). The term $\left[5 E_{V-I}(d)-A_{V}(d)\right]$ describes the interstellar extinction and it is not directly measurable, it rather depends on the adopted model. However, the parameter

$$
\mu_{0} \equiv V-5(V-I)-1.0,
$$

can be calculated for every star from its measured color and magnitude. If there was no extinction we would have $\mu=\mu_{0}$. We shall analyze the distribution of stars as a function of the parameter $\mu_{0}$.

To minimize the contribution of the numerous bulge stars we consider only the region of the CMD clearly dominated by disk stars and selected according to the following three inequalities:

$$
V<19.0, \quad V-I<1.4, \quad(V-I)+0.15(V-19.0)<1.1 .
$$

All stars observed in nine fields in Baade's Window that satisfied the inequalities (16) were counted in bins of $\Delta \mu_{0}=0.2$. The result appears in Figures 5, and 7, where it is compared to several theoretical models described in detail below.

The distributions expected in the standard B\&S model (solid line) and the KDF (Kent et al. 1991) model with the changing disk scale height (dotted line) combined with the Arp-like (1965) extinction law are shown in Figure 5. For $A_{V, G B}=1.5$ these are the distributions shown in the color-magnitude diagrams presented in Figures 3 and 4. It is clear that neither model fits the data well, but the variable scale height model looks somewhat better than the one with the constant scale height.

The most dramatic feature in the observed distribution is the large drop in the number density of stars around $\mu_{0} \approx 10.7$. We introduce the following measure of this drop:

$$
\Delta \log N \equiv \log N_{1}-\log N_{2},
$$


where $N_{1}$ and $N_{2}$ are the number of stars observed in the ranges $9.9 \leq \mu_{0}<10.5$ and $11.1 \leq \mu_{0}<11.7$, respectively. We made an attempt to account for the large value of $\Delta \log N$ by varying the distribution of the interstellar extinction with distance. We introduced the following formula

$$
\begin{array}{lcc}
A_{V}=0 & \text { for } & d<d_{1}, \\
A_{V}=A_{V, G B} \times\left(d-d_{1}\right) /\left(d_{2}-d_{1}\right) & \text { for } & d_{1}<d<d_{2}, \\
A_{V}=A_{V, G B} & \text { for } & d_{2}<d,
\end{array}
$$

where $A_{V, G B}, d_{1}$ and $d_{2}$ are the three adjustable parameters. For every value of $A_{V, G B}$ we varied $d_{1}$ and $d_{2}$ so as to maximize the value of $\Delta \log N$. The result is shown in Fig. 6 for the KDF and B\&S disk models together with the observed value of $\Delta \log N$.

We selected two models indicated with large dots in Fig. 6. These correspond to $\left(A_{V, G B}, d_{1}, d_{2}\right)$ equal to $(1.9 \mathrm{mag}, 1 \mathrm{kpc}, 3 \mathrm{kpc})$ and $(2.6 \mathrm{mag}, 1 \mathrm{kpc}, 3 \mathrm{kpc})$ for the $\mathrm{KDF}$ and B\&S models, respectively. The distributions of $\log N$ versus $\mu_{0}$ are shown in Figure 7 . It is clear that even though the new models have a large drop in the number of stars near $\mu_{0}=10.7$ they also have a very steep rise around $\mu_{0}=9.5$, unlike the observed distribution.

There are two outstanding features in the distribution of the Galactic disk stars as observed in Baade's Window that seem to be impossible to explain with any of the models. First, the number of stars observed in the distance range $0<d<2.5 \mathrm{kpc}$ is approximately a factor of 2 larger than expected. This has already been noticed by Rodgers et al. (1986) and by Terndrup (1988). Second, there is a very sharp drop in the number of observed stars beyond $2.5 \mathrm{kpc}$. It is intriguing that this is the distance at which there is the Sagittarius spiral arm (Oort, Kerr \& Westerhout 1958; Mihalas \& Binney 1981, p. 248, and references therein). It is natural to have a concentration of young star to a spiral arm. However, the most of the stars so clearly observed by the OGLE to form a narrow main sequence in Figure 2 have the absolute magnitude $4<M_{V}<7$. Such stars have the main sequence life time of many billion years. It is reasonable to expect that the star formation rate within the galactic disk has not increased dramatically over the last $10^{8}-10^{9}$ years, or so. Hence, the vast majority of those stars must be very old. An alternative would require the star formation rate at $d \sim 2 \mathrm{kpc}$ to be suppressed over billions of years until the very recent time. We consider this alternative to be unreasonable.

It is surprising to find old stars concentrated to a spiral arm, but it is not unprecedented: there were reports of a similar phenomenon in the spiral galaxy M51, and elsewhere, based on the near infrared surface photometry (Rix \& Rieke 1993, and references therein). Ours is the first indication of such a concentration based on the direct observations of the faint and old main sequence stars. 
We have fully explored two leading models of the Galaxy but we cannot prove beyond any doubt that there is no other explanation for the observed distribution of disk stars except for a concentration in the Sagittarius arm. We have found that the large drop in the observed number of stars beyond $d \approx 2.5 \mathrm{kpc}$ cannot be explained with the total interstellar extinction $A_{V} \approx 1.5$ mag. A reasonable drop could be obtained for $A_{V} \approx 1.9$ mag, and the variable disk scale height, but such a model creates also a large jump in the number of stars near $\mu_{0}=9.5$, a feature that is not observed. In the models with constant disk scale height the total extinction required to account for the large drop in the number of stars at $\mu=10.7$ is far too large to be acceptable $\left(A_{V, G B}=2.6\right)$, and they also create a problem with the jump at $\mu_{0}=9.5$.

At this time we can offer no alternative to our conclusion that there is a dramatic decline in the number of disk main sequence star on the far side of the Sagittarius spiral arm.

There is another intriguing feature in the color-magnitude diagram: there are more red clump stars in the Galactic bulge than there are red giants as defined with the inequalities (1) and (2). This implies that the population cannot be made of very old stars only, with the age $\sim 10^{10}$ years, as in that case there would be many more red giants than red clump stars, as is the case in the very old open cluster NGC 6791 (Kałużny \& Udalski 1992). In a somewhat younger population the stars spend more time in the red clump region and less time in the red giant region, below the red clump. This phenomenon is well documented by the color-magnitude diagrams of intermediate age clusters (Breger 1982; Anthony-Twarog et al. 1990; Paez et al. 1990), as well as by the theoretical models (Castellani et al. 1992; Schuller et al. 1992). A similar effect: the age dependence of the ratio of the number of red clump stars to the number of brighter red giant stars has already been pointed out by Barbaro \& Pigatto (1984). The presence of an age spread in the Galactic bulge has already been noticed (cf. Frogel 1988; Rich 1991, 1992; Holtzman 1993 et al.; and references therein), mostly using the bright red giants or the main sequence turn-off as the age indicators. As far as we know the red clump statistics has never been exploited for this purpose. A quantitative assessment of the age spread is beyond the scope of this paper, but the readers can use our color-magnitude diagram for this purpose (available by ftp - see below).

We have discovered yet another feature in the color-magnitude diagram that is probably related to a similar feature reported by Ortolani et al. (1992) in their field at $(l, b) \approx\left(14^{\circ},-1^{\circ}\right)$. Figure 8 shows a sequence of stars between two dashed lines that extends upwards from the bulge red clump. These lines correspond to the intrinsic colors $(V-I)_{0}=0.8$ and $(V-I)_{0}=1.28$, and the horizontal solid lines correspond to $M_{V}=1.2$ 
at the distance of 1, 2, 4, and $8 \mathrm{kpc}$. Arp's (1965) extinction law is again adopted. The parameters $\left[M_{V},(V-I)_{0}\right]=(1.2,1.28)$ correspond to the peak density of the stars in the red clump of the Galactic bulge (cf. Table 1). It is likely that the sequence of stars present in Figure 8 between the distances of 1 and $4 \mathrm{kpc}$ is made of the disk red clump stars. There are enough of them to notice, but not enough of them to study their CMD morphology.

The distribution of stars in the color-magnitude diagram as observed by OGLE in Baade's Window is available over the computer network using anonymous ftp on astro.princeton.edu. Login as ftp, use your name as a password. Change directory to $\mathrm{bp} / \mathrm{spiral}$. The file read.me contains a list of the necessary files and instructions how to retrieve the data.

We are most grateful to Dr. N. D. Tyson for his many helpful comments and ideas and his careful reading of the draft of this paper. We also acknowledge discussions with Drs. J. P. Ostriker and D. N. Spergel. We also acknowledge a very important comment by the referee, which made us look more quantitatively at the effects of interstellar extinction on the apparent distribution of the stars in the color - magnitude diagram. This project was supported with the NSF grants AST 9216494 and AST 9216830 and Polish KBN grants No 2-1173-91-01 and BST438A/93. 


\section{REFERENCES}

Anthony-Twarog, B. J., Twarog, B. A., Kałużny, J., \& Shara, M. M. 1990, AJ, 99, 1504

Arp, H. 1965, ApJ, 141, 43

Bahcall, J. N. 1986, ARA\&A, 24, 577

Bahcall, J. N., \& Soneira, R. M. 1980, ApJS, 44, 73 (B\&S)

Barbaro, G., \& Pigatto, L. 1984, A\&A, 136, 355

Breger, M. 1982, ApJ, 263, 199

Castellani, V., Chieffi, A. \& Straniero, O. 1992, ApJS, 78, 517

Dean, J. F., Warren, P. R., \& Cousins, A. W. J. 1978, MNRAS, 183, 569

Frogel, J. A. 1988, ARA\&A, 26, 51

Holtzman, J. A., Light, R. M., Baum, W. A., Worthey, G., Faber, S. M., Hunter, D. A., O’Neil, E. J. Jr., Kreidl, T. J., Groth, E. J., \& Westphal, J. A. 1993, AJ, 106, 1826

Kałużny, J., \& Udalski, A. 1992, Acta Astron., 42, 29

Kent, S. M., Dame, T. M., \& Fazio, G. 1991, ApJ, 378, 131

Luyten, W. J. 1968, MNRAS, 139, 221

Mihalas, D., \& Binney, J. 1981, Galactic Astronomy, (San Francisco: Freeman)

Oort, J. H., Kerr, F. T., \& Westerhout, G. 1958, MNRAS, 118, 379

Ortolani, S., Bica, E., \& Barbuy, B. 1992, The Messenger, 68, 54

Paez, E., Martinez Roger, C., Arribas, S., Straniero, O., Caputo, F., \& Castellani, V. 1990, Ap. Space Sci., 169, 41

Prosser, C. F., Stauffer, J., \& Kraft, R. P. 1991, AJ, 101, 1361

Rich, R. M., 1991, in Stellar Populations, IAU Symposium 149, eds. A. Renzini, B. Barbuy, (Dordrecht: Kluwer Academic)

Rich, R. M., 1992, in The Center, Bulge, and Disk of the Milky Way, ed. L. Blitz, (Dordrecht: Kluwer Academic)

Rix, H.-W., \& Rieke, M. J. 1993, ApJ, 418, 123

Rodgers, A. W., Harding, P., \& Ryan, S. 1986, AJ, 92, 600

Schuller, G., Schaerer, D., Meynet, G., \& Maeder, A. 1992, A\&AS, 96, 269

Szymański, M., \& Udalski, A. 1993, Acta Astron., 43, 91 
Terndrup, D. M. 1988, AJ, 96, 884

Tyson, N. D., 1991, Ph. D. Thesis, Columbia University

Udalski, A., Szymański, M., Kałużny, J., Kubiak, M., \& Mateo, M. 1992, Acta Astron., 42, 253

Udalski, A., Szymański, M., Kałużny, J., Kubiak, M., \& Mateo, M. 1993a, Acta Astron., 43,69

Udalski, A., Szymański, M., Kałużny, J., Kubiak, M., Krzemiński, W., Mateo, M., Preston, G. W., \& Paczyński, B. 1993b, Acta Astron., 43, 289

Walker, A. R. 1985, MNRAS, 213, 889

Walker, A. R., \& Mack, P. 1986, MNRAS, 220, 68

Walker, A. R., \& Terndrup, D. M. 1991, ApJ, 378, 119

Wielen, R. 1974, Highlights of Astronomy, Vol. 3, ed. G. Contopoulos (Dordrecht: Reidel) 


\section{FIGURE CAPTIONS}

Fig. 1. - The $V-I$ color-magnitude diagram for stars in the central of the nine Baade's Window fields of the OGLE experiment (Udalski et al. 1993a). The five dashed lines show the approximate location of the Pleiades main sequence (Walker 1985) at the distance of 0.5, 1, 2, 4, and $8 \mathrm{kpc}$, with the interstellar reddening adopted following Arp (1965). The vast majority of the stars are in the Galactic bulge. The disk stars are concentrated along the main sequence line corresponding to the distance of $2 \mathrm{kpc}$.

Fig. 2.- The $V-I$ color-magnitude diagram for stars in all nine Baade's Window fields of the OGLE experiment (Udalski et al. 1993a). As in Figure 1, the five dashed lines show the approximate location of the Pleiades main sequence at the distance of $0.5,1,2,4$, and 8 kpc, with the interstellar reddening adopted following Arp (1965). Also shown are four solid lines that correspond to the disk main sequence stars of the absolute visual magnitude and the unreddened $V-I$ color: $\left[M_{V},(V-I)_{0}\right]=(1.0,0.0),(3.0,0.2),(5.0,0.4)$, and $(7.0,0.6)$. Almost all stars are in the Galactic disk, and about $90 \%$ of them are closer than $3 \mathrm{kpc}$.

Fig. 3.- The $V-I$ color-magnitude diagram for the Galactic disk stars as expected according to the standard model (Bahcall 1986, Bahcal \& Soneira 1980). This Monte-Carlo simulation was done for the field of $\left(40^{\prime}\right)^{2}$ centered on Baade's Window. As in Fig. 1 the five dashed lines show the approximate location of the Pleiades main sequence at the distance of $0.5,1$, 2, 4, and $8 \mathrm{kpc}$, with the interstellar reddening adopted following Arp (1965) and described in section 2. Also shown are four solid lines corresponding to the disk main sequence stars of the absolute visual magnitude and the unreddened $V-I$ color: $\left[M_{V},(V-I)_{0}\right]=(1.0,0.0)$, $(3.0,0.2),(5.0,0.4)$, and $(7.0,0.6)$. The most of the stars in this model are beyond $3 \mathrm{kpc}$.

Fig. 4.- The same as Fig. 3 but for a model with the disk scale height decreasing towards the Galactic center (Kent et al. 1991, cf. eq. 13).

Fig. 5. - The distribution of the number of Galactic disk stars in Baade's Window, N, as a function of distance modulus $\mu_{0}$ (cf. eq. 15) is shown. The error bars correspond to $N^{-1 / 2}$, where $N$ is the number of stars that satisfy the inequalities (16) per bin of $0.2 \mathrm{mag}$. The solid lines represent the standard model (B\&S, Bahcall \& Soneira 1980), while the dotted lines correspond to the model with the disk scale height decreasing towards the Galactic center (KDF, Kent et al. 1991, cf. eq. 13), with the interstellar extinction adopted following Arp (1965, cf. eqs. 3 and Table 1). with $A_{V, G B}=1.5$ and $A_{V, G B}=1.9$, as indicated. 
Fig. 6. - The variation of the largest value of the $\Delta \log N \equiv \log N_{1}-\log N_{2}$ parameter (cf. eq. 17) with the total extinction towards the Galactic Bulge $A_{V, G B}$ is shown for the KDF (Kent et al. 1991 ) and B\&S (Bahcall and Soneira 1986) disk models with the solid and short dash lines, respectively. The observed value of $\Delta \log N$ is shown with a horizontal long dash line. The distributions corresponding to the two large dots are shown in Fig. 7.

Fig. 7.- The same as Fig. 5 but corresponding to the two extreme interstellar extinction laws (cf. eqs. 18 and discussion) indicated in Fig. 6 with the two large dots. The KDF (Kent et al. 1991 ) and B\&S (Bahcall and Soneira 1986) disk models are shown with the solid and short dash lines, respectively.

Fig. 8. - The $V-I$ color-magnitude diagram for stars in all nine Baade's Window fields of the OGLE experiment (Udalski et al. 1993a). The five dashed lines show the approximate location of the Pleiades main sequence at the distance of $0.5,1,2,4$, and $8 \mathrm{kpc}$, with the interstellar reddening adopted following Arp (1965). Also shown are two dashed lines connected with four horizontal solid lines. These solid lines correspond to the location of the red clump stars at the distance of $1,2,4$, and $8 \mathrm{kpc}$.

Table 1. Parameters for the nine Baade's Window fields

\begin{tabular}{ccccccc}
\hline \hline field & $l$ & $b$ & $\Delta(V-I)_{r g}$ & $\Delta(V-I)_{r c}$ & $A_{V, G B}$ & $V_{V-I}$ \\
\hline BW1 & 1.09 & -3.60 & -0.04 & +0.01 & 1.46 & 12.42 \\
BW2 & 0.71 & -3.81 & +0.07 & +0.07 & 1.68 & 12.37 \\
BW3 & 0.92 & -4.19 & +0.02 & +0.07 & 1.62 & 12.38 \\
BW4 & 1.30 & -3.98 & +0.01 & +0.00 & 1.51 & 12.32 \\
BW5 & 0.90 & -3.71 & +0.12 & +0.11 & 1.80 & 12.35 \\
BW6 & 0.82 & -4.00 & +0.06 & +0.09 & 1.70 & 12.32 \\
BW7 & 1.11 & -4.08 & +0.05 & +0.02 & 1.59 & 12.34 \\
BW8 & 1.20 & -3.79 & -0.02 & -0.04 & 1.42 & 12.37 \\
BWC & 1.01 & -3.89 & +0.00 & +0.00 & 1.50 & 12.37 \\
\hline
\end{tabular}

